\title{
On an Isospectral Lie-Poisson System and Its Lie Algebra
}

Anthony M. Bloch and Arieh Iserles

\author{
${ }^{1}$ Department of Mathematics \\ University of Michigan \\ Ann Arbor, MI 48109, USA \\ abloch@umich.edu \\ ${ }^{2}$ Department of Applied Mathematics and Theoretical Physics \\ Centre for Mathematical Sciences \\ University of Cambridge \\ Wilberforce Road, Cambridge CB3 0WA, England \\ A.Iserles@damtp.cam.ac.uk
}

\section{Dedicated to Steve Smale on His 75th Birthday}

\begin{abstract}
In this paper we analyze the matrix differential system $X^{\prime}=\left[N, X^{2}\right]$, where $N$ is skew-symmetric and $X(0)$ is symmetric. We prove that it is isospectral and that it is endowed with a Poisson structure, and we discuss its invariants and Casimirs.

Formulation of the Poisson problem in a Lie-Poisson setting, as a flow on a dual of a Lie algebra, requires a computation of its faithful representation. Although the existence of a faithful representation, assured by the Ado theorem and a symbolic algorithm, due to de Graaf, exists for the general computation of faithful representations of Lie algebras, the practical problem of forming a "tight" representation, convenient for subsequent analytic and numerical work, belongs to numerical algebra. We solve it for the Poisson structure corresponding to the equation $X^{\prime}=\left[N, X^{2}\right]$.
\end{abstract}

Date received: February 2, 2005. Final version received: August 23, 2005. Date accepted: August 25, 2005. Communicated by Felipe Cucker and Peter Olver. Online publication: January 5, 2006. AMS classification: Primary: 58J53; Secondary: 17B80, 37K30.

Key words and phrases: Isospectral flows, Poisson system, Lie algebra, Faithful representations 


\section{Introduction}

The subject matter of this paper is the matrix system of ordinary differential equations,

$$
X^{\prime}=\left[N, X^{2}\right], \quad t \geq 0, \quad X(0)=X_{0} \in \operatorname{Sym}(n),
$$

where $N \in \mathfrak{s} o(n)$ is given. Here $\operatorname{Sym}(n)$ and $\mathfrak{s} o(n)$ denote the symmetric space of real $n \times n$ symmetric matrices and the Lie algebra of real $n \times n$ skew-symmetric matrices, respectively. It is easy to observe (and will be formally proved in the sequel) that $X(t) \in \operatorname{Sym}(n)$ for all $t \geq 0$.

Our interest in the system (1.1) is motivated by four reasons. First, it is trivial to verify that (1.1) can be rewritten in the form

$$
X^{\prime}=[N, X] X+X[N, X], \quad t \geq 0, \quad X(0)=X_{0} \in \operatorname{Sym}(n),
$$

where $[A, B]=A B-B A$ is the standard matrix commutator. Since $[N, X] \in$ $\operatorname{Sym}(n)$ for $N \in \mathfrak{s o}(n), X \in \operatorname{Sym}(n)$, the system (1.2) is a special case of a congruent flow

$$
X^{\prime}=A(X) X+X A^{\top}(X), \quad t \geq 0, \quad X(0)=X_{0} \in \operatorname{Sym}(n),
$$

where $A: \operatorname{Sym}(n) \rightarrow \mathrm{M}(n)$ is sufficiently smooth: here $\mathrm{M}(n)$ denotes the set of real $n \times n$ matrices. It is easy to verify that $X(t)=V(t) X_{0} V^{\top}(t)$, where $V^{\prime}=A\left(V X_{0} V^{\top}\right) V, V(0)=I$. In other words, the solution of (1.1) is an outcome of the general linear group $\operatorname{GL}(n)$ acting on $\operatorname{Sym}(n)$ by congruence. This implies the presence of a number of invariants: the signature of $X(t)$ is constant, and so is the angular field of values, $F^{\prime}(X)=\left\{\boldsymbol{y}^{*} X \boldsymbol{y}: \boldsymbol{y} \in \mathrm{C}^{n} \backslash\{\boldsymbol{0}\}\right\}$ (Horn and Johnson [14]).

Having said so, action by congruence per se is not very interesting and systems (1.3) have never attracted much attention. However, intriguingly, the differential system (1.1) is subject to another group action: it is acted on by the special orthogonal group $\mathrm{SO}(n)$ by similarity. Specifically, letting $B(X)=N X+X N$, we can write it in the form

$$
X^{\prime}=[B(X), X], \quad t \geq 0, \quad X(0)=X_{0} \in \operatorname{Sym}(n) .
$$

Any system of the form (1.4), where $B(X): \operatorname{Sym}(n) \rightarrow \mathfrak{s} o(n)$ (as it does in our case) is isospectral: the eigenvalues of $X(t)$ are invariant. The underlying reason, which we have already indicated, is an $\mathrm{SO}(n)$ action by similarity,

$$
X(t)=Q(t) X_{0} Q^{-1}(t), \quad t \geq 0,
$$

where $Q \in \mathrm{SO}(n)$ is the solution of

$$
Q^{\prime}=B\left(Q X_{0} Q^{-1}\right) Q, \quad t \geq 0, \quad Q(0)=I .
$$

Note, incidentally, that, by virtue of orthogonality, $Q^{-1}=Q^{\top}$, therefore an isospectral flow is a special case of congruent flow. Having said so, isospectral flows 
have received much greater attention than their congruent counterparts, since their invariants are considerably more interesting and feature in a number of important applications,

- The Toda lattice equations of near-neighbor interaction between unit-mass particles can be translated to this form. This is an important tool in their analysis (Flaschka [12]; Toda [21]).

- The familiar QR method for the computation of matrix eigenvalues can be interpreted as sampling a specific isospectral flow at unit intervals (Symes [20]). This insight into the connection between processes in numerical linear algebra and differential flows is fundamental and has spawned much further research (Deift, Nanda, and Tomei [10]; Watkins [23]; Chu [7]).

- The identification of linear-algebraic processes with differential flows has led to new algorithms for several important problems. An excellent example is the work of Chu [6] on the inverse eigenvalue problem for symmetric Toeplitz matrices. Another relevant reference is Bloch, Brockett, and Ratiu [3].

- Letting $B(X)=[N, X]$, where $N \in \operatorname{Sym}(n)$, leads to the double-bracket flows, which have attracted a great deal of attention in the last decade (Brockett [5]; Chu and Driessel [8]; Bloch et al. [3]; Bloch, Brockett, and Crouch [2]; Bloch and Iserles [1]).

- The structure of the matrix $N$ plays a key role in the theory and applications of double-bracket flows. In particular, it assumes a special diagonal form in generalized Toda flows (Bloch et al. [3]) and takes various other forms in linear programming and related problems (Brockett [5]). It may also be time dependent in certain applications of this theory to computing. This is a significant point to emphasize, since it might be of interest to consider special forms of $N$ in (1.1) and eventually time-dependent $N$ and $N=N(X)$. We do not pursue this issue further in this paper.

To recap, the differential system (1.1) is evolving under two distinct group actions. This makes it fairly unusual (although by no means unique) and worthy of further analysis.

Another aspect of equations (1.1) that renders them interesting is that they are, in a sense, dual to the generalized rigid body equations

$$
M^{\prime}=[\Omega, M], \quad t \geq 0, \quad M(0) \in \mathfrak{s} o(n),
$$

where $M=\Omega J+J \Omega, J \in \operatorname{Sym}(n)$ (therefore, $\Omega \in \mathfrak{s} o(n)$ ) (Manakov [16]). This point will be further elaborated on in Section 3.

Our final motivation for the study of (1.1) originates in the apparent behavior of their solutions. Thus, for example, we have computed numerically the solution of the system for

$$
N=\left[\begin{array}{rrr}
0 & 1 & 0 \\
-1 & 0 & 1 \\
0 & -1 & 0
\end{array}\right]
$$



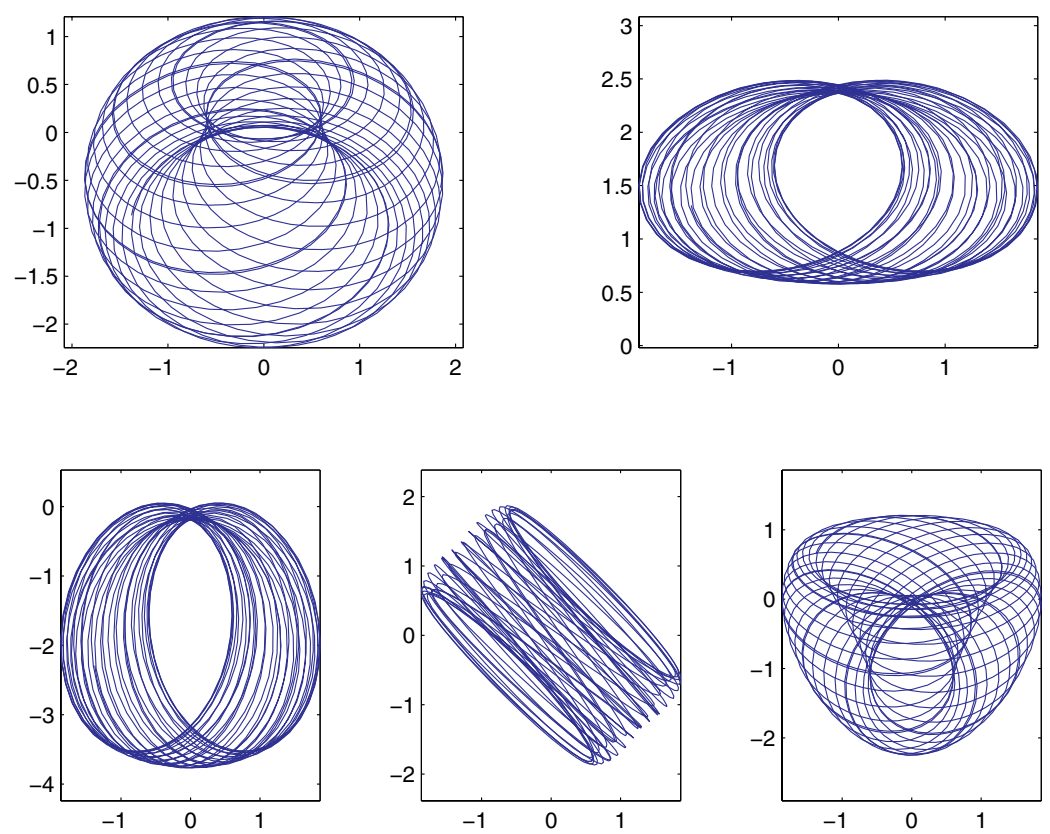

Fig. 1. The phase portraits $\left(x_{1,2}, x_{k, l}\right)$ for $(k, l)=(1,1),(1,3),(2,2),(2,3),(3,3)$, with a random initial condition.

In Figure 1 we display the phase portraits of the solution. Clearly, the solution displays a great deal of regularity and apparently it evolves on invariant tori. Similar behavior is obtained for other matrices $N$, also in a larger number of dimensions.

It is fair to say that this kind of regular behavior seldom arises at random. Typically it is an indication of a deeper structure, often either Hamiltonian or related to Hamiltonian. The purpose of this paper is to probe and understand this structure and this is discussed further in Bloch, Iserles, Marsden, and Ratiu [4].

In Section 2 we prove that the matrix system (1.1) can be, at the first instance, written as a set of almost-Poisson equations,

$$
\boldsymbol{x}^{\prime}=S(\boldsymbol{x}) \nabla H(\boldsymbol{x}), \quad t \geq 0, \quad \boldsymbol{x}(0)=\boldsymbol{x}_{0} \in \mathrm{R}^{(1 / 2) n(n+1)} .
$$

Here $\boldsymbol{x}$ is the upper-triangular portion of the symmetric matrix $X$, "stretched" as a vector, the smooth function $S \in \mathfrak{s o}\left(\frac{1}{2} n(n+1)\right)$ is linear and homogeneous and the Hamiltonian $H$ is smooth and nonnegative. Moreover, we verify that the structure matrix $S$ obeys the Jacobi identity: for every $p, q, r=1,2, \ldots, \frac{1}{2} n(n+1)$ it is true that

$$
\sum_{k=1}^{(1 / 2) n(n+1)}\left[\frac{\partial S_{p, q}}{\partial x_{k}} S_{k, r}+\frac{\partial S_{q, r}}{\partial x_{k}} S_{k, p}+\frac{\partial S_{r, p}}{\partial x_{k}} S_{k, q}\right]=0
$$


Therefore

$$
\{f, g\}=(\nabla f)^{\top} S(\nabla g)
$$

defines a Poisson bracket and (1.6) is a Poisson system. This has important implications, which we discuss briefly and which will be discussed more comprehensively elsewhere.

Section 3 is devoted to a preliminary investigation of a critical aspect of Poisson flows, focussing on their invariants and Casimirs. We prove that (1.1) has $\approx \frac{1}{4} n^{2}$ invariants and at least two Casimirs: $\operatorname{det} X$ and $1^{\top} N X 1$. A forthcoming paper discusses the integrability of (1.1) in detail, identifies all the Casimirs in two relevant Poisson structures, and proves involutivity of the relevant integrals of motion (Bloch et al. [4]).

The main computational content of the paper is Section 4. A Poisson system can be written in a Lie-Poisson form, as a flow over an orbit in a dual $\mathfrak{g}^{*}$ of a Lie algebra $\mathfrak{g}$ which is determined by the elements of the structure matrix $S$. Specifically, recalling that $S$ is linear and homogeneous in $\boldsymbol{x}$, there exist structure constants $c_{p, q}^{r}, p, q, r=1,2, \ldots, m$, where $m=\frac{1}{2} n(n+1)$, such that

$$
S_{p, q}(\boldsymbol{x})=\sum_{r=1}^{m} c_{p, q}^{r} x_{r}, \quad p, q=1,2, \ldots, m .
$$

Skew-symmetry of $S$ is equivalent to

$$
c_{p, q}^{r}+c_{q, p}^{r}=0, \quad p, q, r=1, \ldots, m,
$$

while the Jacobi identity (1.7) is equivalent to

$$
\sum_{i=1}^{m}\left(c_{p, q}^{i} c_{i, r}^{s}+c_{q, r}^{i} c_{i, p}^{s}+c_{r, p}^{i} c_{i, q}^{s}\right)=0, \quad p, q, r, s=1,2, \ldots, n .
$$

Now, (1.8) and (1.9) are precisely the conditions for the $\left\{c_{p, q}^{r}\right\}$ to form structure constants of a finite-dimensional Lie algebra (Olver [18]). Let us denote this algebra by $\mathfrak{g}$ and assume that $\left\{E_{1}, E_{2}, \ldots, E_{m}\right\}$ is its basis. Moreover, let us suppose that $\left\{F_{1}, F_{2}, \ldots, F_{m}\right\}$ form the basis of the dual $\mathfrak{g}^{*}$ (the linear space of linear functionals acting on $\mathfrak{g})$ and that $\left\langle F_{k}, E_{l}\right\rangle=\delta_{k, l}, k, l=1,2, \ldots, m$, where

$$
\langle Y, Z\rangle=\operatorname{tr}\left(Y^{\top} Z\right)
$$

and $\delta_{k, l}$ is the familiar Kronecker delta. The following construction is general to all Poisson systems (Marsden and Ratiu [17]). We associate with $\boldsymbol{x}$, the solution of (1.6), an element of $\mathfrak{g}^{*}$, namely

$$
Y(t)=\sum_{k=1}^{m} x_{k}(t) F_{k} .
$$


With minor abuse of notation, we let $H(Y)=H(x)$. Then (1.6) is equivalent to

$$
Y^{\prime}=-\operatorname{ad}_{H^{\prime}(Y)}^{*} Y, \quad t \geq 0, \quad Y(0)=\sum_{k=1}^{m} x_{k}(0) F_{k},
$$

where ad* is the adjoint operator in $\mathfrak{g}^{*}$ and $H^{\prime}(Y)=\left(\partial H(Y) / \partial Y_{p, q}\right)_{p, q=1, \ldots, m}$.

The formulation of a Poisson system as a flow in $\mathfrak{g}^{*}$ is advantageous for a number of analytic and numerical reasons (Engø and Faltinsen [11]; Lewis and Simo [15]; Marsden and Ratiu [17]). However, practical implementation of (1.10) requires a representation of $F_{1}, F_{2}, \ldots, F_{m}$ by matrices. In principle, this can be done since, by the Ado theorem, every finite-dimensional Lie algebra possesses a faithful representation (Varadarajan [22]). Yet, the Ado theorem falls short of providing such a representation in an explicit form. In principle, it is possible to use symbolic algebra to this end: in a beautiful paper, de Graff [9] demonstrated how to render Ado's original proof into a constructive algorithm. Yet, this falls short of our requirements. First, the algorithm delivers the $E_{k}$ 's, but not a biorthogonal basis of $\mathfrak{g}^{*}$. Second, and more importantly, the size of matrices in de Graff's algorithm increases exponentially with $m$, while practical work with (1.10) requires either a minimal representation or, at the very least, one which is fairly small. In Section 4 we introduce a numerical, linear-algebraic algorithm that performs this task for our specific Lie-Poisson flow and produces matrices of size $(2 n-2) \times(2 n-2)$.

Assuming without significant loss of generality that $n$ is even, any $N \in \mathfrak{s} o(n)$ can be reduced by a real similarity transformation to a tridiagonal matrix with $2 \times 2$ skew-symmetric blocks along the diagonal. Since we can subject $X_{0}$ to a similar transformation, we could have assumed at the outset that $N$ is of this form. Occasionally this leads to simplification, often it does not. Our impression is that, overall, it is more convenient to assume that $N$ is a general $\mathfrak{s} o(n)$ matrix, but more detailed examination of specific aspects of (1.1) might well benefit from such a similarity transformation (Bloch et al. [4]).

Inasmuch as this is a paper on a specific Lie-Poisson system and the representation of "its" Lie algebra, we address ourselves to a particular instance of a considerably more general problem. Given structure constants $\left\{c_{p, q}^{r}\right\}$ that obey (1.8) and (1.9), hence being within the conditions of the Ado theorem, determine a small faithful representation of the underlying free Lie algebra. This is, essentially, a problem of numerical algebra which, to our knowledge, has been never addressed by numerical algebraists. A major goal of the present paper is to demonstrate that, at least in one case, this problem is tractable. The general case remains as a substantive challenge.

\section{From a Differential Flow to a Poisson System}

An ordinary differential system is said to be almost Poisson if it is of the form

$$
\boldsymbol{x}^{\prime}=S(\boldsymbol{x}) \nabla H(\boldsymbol{x}), \quad t \geq 0, \quad \boldsymbol{x}(0)=\boldsymbol{x}_{0} \in \mathrm{R}^{m},
$$


where $H: \mathrm{R}^{m} \rightarrow \mathrm{R}$ (the Hamiltonian) and the linear, homogeneous matrix function $S: \mathrm{R}^{m} \rightarrow \mathfrak{s} o(m)$ (the structure matrix) are sufficiently smooth. The constants $\left\{c_{p, q}^{r}\right\}_{p, q, r=1, \ldots, m}$ such that

$$
S_{p, q}(\boldsymbol{x})=\sum_{r=1}^{m} c_{p, q}^{r} x_{r}, \quad p, q=1, \ldots, m
$$

are called the structure constants of (2.1) with respect to a basis of $\mathrm{R}^{m}$ implicitly defined by the representation of $\boldsymbol{x}$. Note that

$$
c_{p, q}^{r}+c_{q, p}^{r}=0, \quad p, q, r=1, \ldots, m .
$$

Our contention is that (1.1) is almost Poisson. Writing (1.1) in a coordinate-bycoordinate fashion, it becomes

$$
x_{p, q}^{\prime}=\sum_{r=1}^{n} \sum_{s=1}^{n}\left(n_{p, r} x_{r, s} x_{q, s}-n_{r, s} x_{p, r} x_{q, s}-n_{r, s} x_{p, s} x_{q, r}+n_{q, r} x_{p, s} x_{r, s}\right)
$$

for $p, q=1, \ldots, n$. By virtue of $N \in \mathfrak{s} o(n)$, however, once we swap the indices $r$ and $s$ we have

$$
\sum_{r=1}^{n} \sum_{s=1}^{n} n_{r, s} x_{p, s} x_{q, r}=\sum_{s=1}^{n} \sum_{r=1}^{n} n_{s, r} x_{p, r} x_{q, s}=-\sum_{r=1}^{n} \sum_{s=1}^{n} n_{r, s} x_{p, r} x_{q, s} .
$$

Thus, taking into account the symmetry of $X$, we obtain $m=\frac{1}{2} n(n+1)$ equations

$$
\begin{aligned}
x_{p, q}^{\prime}= & \sum_{r=1}^{n}\left(n_{p, r} x_{q, r}+n_{q, r} n_{p, r}\right) x_{r, r} \\
& +2 \sum_{r=1}^{n-1} \sum_{s=r+1}^{n}\left(n_{p, r} x_{q, s}+n_{q, r} x_{p, s}\right) x_{r, s}, \quad(p, q) \in \mathbf{J}_{n},
\end{aligned}
$$

where

$$
\mathrm{J}_{n}=\{(p, q): 1 \leq p \leq q \leq n\} .
$$

Writing (1.1) in an almost-Poisson form, we need to "stretch" $\left\{x_{p, q}\right\}_{(p, q) \in \mathbb{J}_{n}}$ into a vector $\boldsymbol{x} \in \mathrm{R}^{m}$. It is helpful to retain both indices of the elements of $X$, while ordering them lexicographically: for example, for $n=3$ we have $m=6$ and

$$
\boldsymbol{x}=\left[\begin{array}{llllll}
x_{1,1} & x_{1,2} & x_{1,3} & x_{2,2} & x_{2,3} & x_{3,3}
\end{array}\right]^{\top} .
$$

Note that we may occasionally use $x_{p, q}$ with $p>q$, since this makes our presentation more transparent. In that case, of course, $x_{p, q}=x_{q, p}$.

We set

$$
H(\boldsymbol{x})=\frac{1}{2}\|X\|_{\mathrm{F}}^{2}=\frac{1}{2} \sum_{r=1}^{n} x_{r, r}^{2}+\sum_{r=1}^{n-1} \sum_{s=r+1}^{n} x_{r, s}^{2}
$$


hence (2.3) can be rewritten in the form

$$
x_{p, q}^{\prime}=\sum_{r=1}^{n} \sum_{s=r}^{n}\left(n_{p, r} x_{q, s}+n_{q, r} x_{p, s}\right) \frac{\partial H(\boldsymbol{x})}{\partial x_{r, s}}, \quad(p, q) \in \mathbf{J}_{n}
$$

This form looks tantalizingly similar to (2.1), except that letting $S_{(p, q),(r, s)}(\boldsymbol{x})=$ $n_{p, r} x_{q, s}+n_{q, r} x_{p, s}$ does not lead to a skew-symmetric matrix. However, identifying $x_{q, p}=x_{p, q}$ yields

$$
x_{p, q}^{\prime}=x_{q, p}^{\prime}=\sum_{r=1}^{n} \sum_{s=r}^{n}\left(n_{q, r} x_{p, s}+n_{p, r} x_{q, s}\right) \frac{\partial H(\boldsymbol{x})}{\partial x_{r, s}}, \quad(p, q) \in \mathrm{J}_{n} .
$$

We now average the two expressions: the outcome is

$$
x_{p, q}^{\prime}=\frac{1}{2} \sum_{(r, s) \in \mathbb{J}_{n}}\left(n_{p, r} x_{q, s}+n_{p, s} x_{q, r}+n_{q, r} x_{p, s}+n_{q, s} x_{p, r}\right) \frac{\partial H(\boldsymbol{x})}{\partial x_{r, s}}, \quad(p, q) \in \mathrm{J}_{n} .
$$

This is consistent with the almost-Poisson form (2.1), once we define the Hamiltonian $H$ by (2.4) and let

$S_{(p, q),(r, s)}(\boldsymbol{x})=\frac{1}{2}\left(n_{p, r} x_{q, s}+n_{p, s} x_{q, r}+n_{q, r} x_{p, s}+n_{q, s} x_{p, r}\right), \quad(p, q),(r, s) \in \mathbf{J}_{n}$.

If $S$ obeys the Jacobi identity (1.7) (with $\frac{1}{2} n(n+1)$ replaced by $m$ ), the almostPoisson equations (2.1) are said to be a Poisson (or, alternatively, Kostant-KirillovLie-Souriau) system (Marsden and Ratiu [17]). In that case, as already stated in Section 1, the structure constants obey the identities

$$
\sum_{i=1}^{m}\left(c_{p, q}^{i} c_{i, r}^{s}+c_{q, r}^{i} c_{i, p}^{s}+c_{r, p}^{i} c_{i, q}^{s}\right)=0, \quad p, q, r, s=1, \ldots, m
$$

Theorem 1. The system (2.1) with the Hamiltonian (2.4) and the structure matrix (2.5) is Poisson.

Proof. The matrix $S$ is linear and homogeneous in $\boldsymbol{x}$. Moreover, $N \in \mathfrak{s o}(n)$ and $X \in \operatorname{Sym}(n)$ imply at once that $S \in \mathfrak{s} o(m)$. The system is thus almost Poisson.

The structure constants associated with $S$ are

$$
\begin{aligned}
c_{(p, q),(r, s)}^{(k, l)}= & \frac{1}{2}\left[\delta_{(q, s)}^{(k, l)} n_{p, r}+\delta_{(q, r)}^{(k, l)} n_{p, s}+\delta_{(p, s)}^{(k, l)} n_{q, r}+\delta_{(p, r)}^{(k, l)} n_{q, s}\right], \\
& (p, q),(r, s),(k, l) \in \mathrm{J}_{n},
\end{aligned}
$$

where $\delta_{(i, j)}^{(k, l)}$ is the "symmetrized" Kronecker delta,

$$
\delta_{(i, j)}^{(k, l)}= \begin{cases}1, & (i, j)=(k, l) \text { or }(i, j)=(l, k) \\ 0, & \text { otherwise. }\end{cases}
$$


It is possible to prove directly that the above structure constants satisfy the Jacobi condition (2.6). This requires a great deal of fairly tedious algebra. Instead, we follow a (gratefully acknowledged) suggestion of Peter Olver, which identifies the structure constants (2.7) with an unusual Lie algebra. Let $Y, Z \in \operatorname{Sym}(n)$, $N \in \mathfrak{s} o(n)$, and let

$$
[Y, Z]_{N}=Y N Z-Z N Y .
$$

This is a proper Lie bracket, since $[Z, Y]_{N}=-[Y, Z]_{N}$ and

$$
\begin{aligned}
& {\left[X,[Y, Z]_{N}\right]_{N}+\left[Y,[Z, X]_{N}\right]_{N}+\left[Z,[X, Y]_{N}\right]_{N} } \\
&=\{X N(Y N Z-Z N Y)-(Y N Z-Z N Y) N X\} \\
&+\{Y N(Z N X-X N Z)-(Z N X-X N Z) N Y\} \\
&+\{Z N(X N Y-Y N X)-(X N Y-Y N X) N Z\} \\
&= O
\end{aligned}
$$

-hence the Jacobi identity. Since $\operatorname{Sym}(n)$ is a linear space, it follows at once that, once accompanied by the bracket (2.8), it is a Lie algebra, $\mathfrak{h}_{n}(N)$, say.

We now define

$$
H_{p, q}=\frac{1}{2}\left(\boldsymbol{e}_{p} \boldsymbol{e}_{q}^{\top}+\boldsymbol{e}_{q} \boldsymbol{e}_{p}^{\top}\right), \quad(p, q) \in \mathrm{J}_{n},
$$

where $\boldsymbol{e}_{k} \in \mathrm{R}^{n}$ is the $n$th unit vector, and note that $\left\{H_{p, q}:(p, q) \in \mathrm{J}_{n}\right\}$ form a basis of $\operatorname{Sym}(n)$. A straightforward computation confirms that

$$
\begin{aligned}
{\left[H_{p, q}, H_{r, s}\right]_{N}=} & \frac{1}{2}\left(n_{p, r} H_{q, s}+n_{p, s} H_{q, r}+n_{q, r} H_{p, s}+n_{q, s} H_{p, r}\right), \\
& (p, q),(r, s) \in \mathrm{J}_{n},
\end{aligned}
$$

where we identify $H_{p, q}=H_{q, p}$. Therefore, (2.7) are the structure constants associated with the underlying basis. Hence they satisfy (2.6) (with obvious amendments to cater for "double" indices), we deduce the Jacobi identity, and conclude that the system (2.1) is indeed Poisson.

In Section 4 we are concerned with a faithful representation of the Lie algebra associated with the structure constants (2.7). Here we just state the obvious: (2.8) is not a representation, faithful or otherwise: a representation is defined with the standard matrix commutator $[Y, Z]=Y Z-Z Y$. To the contrary! Once we derive a faithful representation of the Lie algebra associated with the structure constants (2.7), motivated by our goal to represent (1.1) as a Lie-Poisson flow, we simultaneously obtain gratis a faithful representation of $\mathfrak{h}_{n}(N)$.

Further discussion of the Poisson structure for the system (1.1) is given in Bloch et al. [4]. 


\section{Invariants}

\subsection{Lax Pairs and Their Consequences}

It is of interest to compute the invariants for the flow of the system (1.1). We bear in mind that, by virtue of the isospectral representation (1.2), we already know that the eigenvalues of $X$ or, alternatively, $\operatorname{tr} X^{k}$ for $k=1,2, \ldots, n-1$, are invariants. This section represents preliminary investigation into the integrability of (1.1) and we mention in passing that, since the completion of this paper, the issue has been settled out comprehensively in Bloch et al. [4].

One way to compute additional invariants is to rewrite the system as a Lax pair with parameter. One can do this in a fashion which is similar to that for the generalized rigid body equations, see Manakov [16].

Theorem 2. Let $\lambda$ be an arbitrary time-independent parameter with values in $R$. The system (1.4) is equivalent to the Lax pair system

$$
(X+\lambda N)^{\prime}=\left[N X+X N+\lambda N^{2}, X+\lambda N\right] .
$$

Proof. The proof is a computation. In particular, we can verify that the coefficient of $\lambda$ on the right-hand side of equation (3.1) is given by

$$
\begin{aligned}
& \lambda\left([N X+X N, N]+\left[N^{2}, X\right]\right) \\
& \quad=\lambda\left(N X N+X N^{2}-N^{2} X-N X N+N^{2} X-X N^{2}\right),
\end{aligned}
$$

which is zero.

We recall from Manakov [16] and Ratiu [19] that the left-invariant generalized rigid body equations on $\mathrm{SO}(n)$ may be written as

$$
M^{\prime}=[M, \Omega], \quad t \geq 0, \quad M(0)=M_{0} \in \mathfrak{s} o(n),
$$

where $Q \in \mathrm{SO}(n)$ denotes the configuration space variable (the attitude of the body), $\Omega=Q^{-1} Q^{\prime} \in \mathfrak{s} o(n)$ is the body angular velocity, and

$$
M=J(\Omega)=\Lambda \Omega+\Omega \Lambda \in \mathfrak{s} o(n)
$$

is the body angular momentum. Here $J: \mathfrak{s o}(n) \rightarrow \mathfrak{s o}(n)$ is the symmetric, positive definite (and hence invertible) operator defined by

$$
J(\Omega)=\Lambda \Omega+\Omega \Lambda
$$

where $\Lambda$ is a diagonal matrix satisfying $\Lambda_{i}+\Lambda_{j}>0$ for all $i \neq j$. For $n=3$ the elements of $\Lambda_{i}$ are related to the standard diagonal moment of inertia tensor $I$ by $I_{1}=\Lambda_{2}+\Lambda_{3}, I_{2}=\Lambda_{3}+\Lambda_{1}, I_{3}=\Lambda_{1}+\Lambda_{2}$. 
In this case the generalized rigid body equations as Lax equations with parameter due to Manakov take the form

$$
\frac{d}{d t}\left(M+\lambda \Lambda^{2}\right)=\left[M+\lambda \Lambda^{2}, \Omega+\lambda \Lambda\right] .
$$

Note the contrast with our setting: in the Manakov case the system matrix $M$ is in $\mathfrak{s o}(n)$ and $\Lambda$ is a symmetric matrix, while in our case $X$ is symmetric and $N \in \mathfrak{s} o(n)$.

For the generalized rigid body the nontrivial coefficients of $\lambda$ in the traces of the powers of $M+\lambda \Lambda^{2}$ then yield the right number of independent integrals in involution to prove integrability of the flow on a generic adjoint orbit of $\operatorname{SO}(n)$ (identified with the corresponding coadjoint orbit).

Similarly in our case the nontrivial coefficients of $\lambda$ in the traces of the powers of $X+\lambda N$ yield conserved quantities of motion:

$$
\operatorname{tr}(X+\lambda N)^{k}=\text { const }, \quad k=1,2, \ldots, n-1, \quad \lambda \in \mathrm{R} .
$$

Therefore, expanding the power and assembling equal powers of $\lambda$,

$$
\begin{gathered}
\operatorname{tr} \sum_{|i|=r} \sum_{|j|=k-r} X^{i_{1}} N^{j_{1}} X^{i_{2}} \cdots X^{i_{s}} N^{j_{s}}=\mathrm{const}, \\
\quad r=1, \ldots, k, \quad k=1, \ldots, n-1 .
\end{gathered}
$$

Here $\boldsymbol{i}$ is a multi-index and $|\boldsymbol{i}|=\sum_{q} i_{q}$. However, since a trace of a matrix equals the trace of its transpose, $X \in \operatorname{Sym}(n)$ and $N \in \mathfrak{s} o(n)$, it is true that

$$
\operatorname{tr} X^{i_{1}} N^{j_{1}} X^{i_{2}} \cdots X^{i_{s}} N^{j_{s}}=(-1)^{|j|} \operatorname{tr} N^{j_{s}} X^{j_{s}} \cdots X^{i_{2}} N^{j_{1}} X^{i_{1}} .
$$

Therefore, if $k-s$ is odd, then, necessarily,

$$
\operatorname{tr} \sum_{|i|=r} \sum_{|j|=k-r} X^{i_{1}} N^{j_{1}} X^{i_{2}} \cdots X^{i_{s}} N^{j_{s}}=0
$$

and only even $k-s$ qualifies as an invariant. Thus, we are left with the invariants

$\operatorname{tr} \sum_{|i|=k-2 r} \sum_{|j|=2 r} X^{i_{1}} N^{j_{1}} X^{i_{2}} \ldots X^{i_{s}} N^{j_{s}}, \quad k=1, \ldots, n-1, r=0,1, \ldots,\left\lfloor\frac{k-1}{2}\right\rfloor$.

Altogether, this results in

$$
\left\lfloor\frac{n}{2}\right\rfloor \times\left\lfloor\frac{n+1}{2}\right\rfloor
$$

invariants. Building upon the results of this subsection, independence and involution of these integrals are addressed in Bloch et al. [4]. 


\subsection{Casimirs}

It is natural to attempt an analysis of Casimirs for our system with our given Poisson structure. Recall that given two smooth functions $f, g: \mathrm{R}^{m} \rightarrow \mathrm{R}$ and the skew-symmetric function $S(y)$ from (2.5), the Poisson bracket is

$$
\{f, g\}=[\boldsymbol{\nabla} f(\boldsymbol{y})]^{\top} S(\boldsymbol{y}) \boldsymbol{\nabla} g(\boldsymbol{y})=\sum_{i=1}^{m} \sum_{j=1}^{m} \frac{\partial f(\boldsymbol{y})}{\partial y_{i}} S_{i, j}(\boldsymbol{y}) \frac{\partial g(\boldsymbol{y})}{\partial y_{j}} .
$$

A Casimir of the Lie-Poisson system (2.1) is a function $C$ which is in involution (with respect to the above Lie bracket) with all other functions on the Poisson manifold (Marsden and Ratiu [17]). According to the Darboux theorem, if $S(\boldsymbol{x})$ is (locally) of constant even rank $m-\alpha$, say, where $\alpha \geq 1$, then there exist $\alpha$ Casimirs, $c_{1}, c_{2}, \ldots, c_{\alpha}$, say, which are themselves in involution. They satisfy

$$
\left\{c_{k}, g\right\}=0 \quad \text { for all smooth } g .
$$

Each Casimir is a first integral of the Lie-Poisson system.

Extensive experimentation with MATLAB, generating a large number of matrices $S$ using random $N \in \mathfrak{s o}(n)$ and $X \in \operatorname{Sym}(n)$, seems to indicate that $\alpha=\lfloor(n+$ 1)/2」. Note that, in that case, $m-\alpha=2\lfloor n / 2\rfloor \times\lfloor(n+1) / 2\rfloor$ is indeed even.

Lemma 3. Suppose that $n \geq 3$ and $X_{0}, N \neq O$, where $O$ is the zero matrix. Then it is true for the system (2.1) with the structure matrix (2.5) that $\alpha \geq 2$.

Proof. We will demonstrate that $\alpha \geq 2$ by singling out two linearly independent eigenvectors $\boldsymbol{v}, \boldsymbol{w} \in \mathrm{R}^{m}$ that correspond to a zero eigenvalue, $S \boldsymbol{v}=S \boldsymbol{w}=0$.

We first assume that both $X$ and $N$ are nonsingular. The elements of the eigenvectors will be denoted by the usual double indices. We first let

$$
v_{p, q}= \begin{cases}\frac{1}{2}\left(X^{-1}\right)_{p, p}, & p=q \\ \left(X^{-1}\right)_{p, q}, & p<q .\end{cases}
$$

Therefore, using (2.5) and exploiting as necessary symmetry of $X$ and $V$ and skew-symmetry of $N$,

$$
\begin{aligned}
(S v)_{p, q} & =\sum_{(r, s) \in \mathbb{J}_{n}} S_{(p, q),(r, s)} v_{r, s} \\
& =\frac{1}{2} \sum_{r=1}^{n} \sum_{s=r}^{n}\left(n_{p, r} x_{q, s}+n_{p, s} x_{q, r}+n_{q, r} x_{p, s}+n_{q, s} x_{p, r}\right) v_{r, s} \\
& =\frac{1}{4} \sum_{r=1}^{n} \sum_{s=1}^{n}\left(n_{p, r} x_{q, s}+n_{p, s} x_{q, r}+n_{q, r} x_{p, s}+n_{q, s} x_{p, r}\right)\left(X^{-1}\right)_{r, s}
\end{aligned}
$$




$$
\begin{aligned}
& =\frac{1}{4}\left[\sum_{r=1}^{n} n_{p, r} \sum_{s=1}^{n} x_{q, s}\left(X^{-1}\right)_{s, r}+\sum_{s=1}^{n} n_{p, s} \sum_{r=1}^{n} x_{q, r}\left(X^{-1}\right)_{r, s}\right. \\
& \left.\quad+\sum_{r=1}^{n} n_{q, r} \sum_{s=1}^{n} x_{p, s}\left(X^{-1}\right)_{s, r}+\sum_{s=1}^{n} n_{q, s} \sum_{r=1}^{n} x_{p, r}\left(X^{-1}\right)_{r, s}\right] \\
& =\frac{1}{4}\left(n_{p, q}+n_{p, q}+n_{q, p}+n_{q, p}\right)=0 .
\end{aligned}
$$

Therefore, indeed, $S \boldsymbol{v}=0$.

Likewise, we set

$$
w_{p, q}= \begin{cases}\frac{1}{2}\left(N^{-1}\right)_{p, p}, & p=q, \\ \left(N^{-1}\right)_{p, q}, & p<q .\end{cases}
$$

The proof of $S \boldsymbol{w}=0$ follows in an identical manner, because each $S_{(p, q),(r, s)}$ is symmetric in $X$ and $N$.

The requirement that $X$ and $N$ are nonsingular can be easily lifted, replacing the inverse by an adjugate matrix, as necessary: because of our assumptions, $\boldsymbol{v}, \boldsymbol{w} \neq 0$. Since $X \in \operatorname{Sym}(n)$ cannot be a multiple of $N \in \mathfrak{s o}(n)$, the vectors $\boldsymbol{v}$ and $\boldsymbol{w}$ are linearly independent. This concludes the proof.

Note that, in a sense, $\boldsymbol{v}$ and $\boldsymbol{w}$ are "dual" to each other: while $\boldsymbol{v}=\boldsymbol{v}(\boldsymbol{x})$, but is independent of $N, \boldsymbol{w}$ is dependent solely on $N$.

The following result identifies two Casimirs of the system (1.1).

Theorem 4. Let $c_{1}(X)=\operatorname{det} X$. Then, for every $(p, q) \in J_{n}$, it is true that

$$
\begin{aligned}
& \frac{1}{c_{1}(X)} \frac{\partial c_{1}(X)}{\partial x_{p, p}}=\left(X^{-1}\right)_{p, p}, \quad k=1,2, \ldots, n, \\
& \frac{1}{c_{1}(X)} \frac{\partial c_{1}(X)}{\partial x_{p, q}}=2\left(X^{-1}\right)_{p, q}
\end{aligned}
$$

and $c_{1}$ is a Casimir of the system (1.1). Likewise,

$$
c_{2}(X)=\frac{1}{2} \mathbf{1}^{\top}(\operatorname{adj} N) X \mathbf{1},
$$

where $\operatorname{adj} N$ is the adjugate matrix of $N$ and is a Casimir of (1.1) for $N \neq O$. (We may replace $\operatorname{adj} N$ by $N^{-1}$ if $N$ is nonsingular.)

Proof. We denote by $A_{p, q}$ a matrix $A$ from which we have excised the $p$ th column and the $q$ th row. Thus,

$$
\left(X^{-1}\right)_{p, q}=(-1)^{p+q} \frac{\operatorname{det} X_{q, p}}{\operatorname{det} X}=(-1)^{p+q} \frac{\operatorname{det} X_{p, q}}{c_{1}(X)} .
$$

Therefore, it is enough to prove that

$$
\frac{\partial c_{1}(X)}{\partial x_{p, p}}=\operatorname{det} X_{p, p}, \quad \frac{\partial c_{1}(X)}{\partial x_{p, q}}=2(-1)^{p+q} \operatorname{det} X_{p, q}, \quad p \neq q .
$$


For $p=l$ the element $x_{p, p}$ appears just once in $X$ and taking the derivative of $c_{1}(X)=\operatorname{det} X$ with respect to $x_{p, p}$ replaces the $p$ th row and the $p$ th column by $\boldsymbol{e}_{p}^{\top}$ and $\boldsymbol{e}_{p}$, respectively. Thus, $\partial \operatorname{det} X / \partial x_{p, p}=\operatorname{det} X_{p, p}$.

For $p \neq q$ the term $x_{p, q}$ appears twice in $X$ and the derivative is thus a sum of two determinants: one when the $p$ th row is replaced by $\boldsymbol{e}_{p}^{\top}$ and the $q$ th column by $\boldsymbol{e}_{q}$ and the other when the $p$ th row and the $q$ th column are replaced by $\boldsymbol{e}_{q}^{\top}$ and $\boldsymbol{e}_{p}$, respectively. Because of symmetry, both determinants equal $(-1)^{p+q} \operatorname{det} X_{p, q}$. This completes the proof.

Consequently, $c_{1}(X)=\operatorname{det} X$ is a Casimir iff

$$
\sum_{r=1}^{n} S_{(p, q),(r, r)}\left(X^{-1}\right)_{r, r}+2 \sum_{r=1}^{n-1} \sum_{s=r+1}^{n} S_{(p, q),(r, s)}\left(X^{-1}\right)_{r, s}=0
$$

for every $(p, q) \in \mathrm{J}_{n}$. This, however, follows at once from the proof of Lemma 3 , once we observe that the partial derivatives of $c_{1}$, scaled by $c_{1}^{-1}(X)$, are precisely the elements of the vector $v$ therein.

The proof that $c_{2}$ is a Casimir follows from the observation that, once we consider the "reduced" system with $m=\frac{1}{2} n(n+1)$, it is true that

$$
\frac{\partial c_{2}(X)}{\partial x_{p, q}}=\left\{\begin{array}{ll}
\frac{1}{2}(\operatorname{adj} N)_{p, p}, & p=q, \\
(\operatorname{adj} N)_{p, q}, & p<q,
\end{array} \quad(p, q) \in \mathbf{J}_{n} .\right.
$$

This, according to Lemma 3, is an eigenvector of $S$ : the proof therein was for $N^{-1}=(\operatorname{det} N)^{-1}$ adj $N$ but, as already remarked, it is equally valid for the adjugate matrix.

Of course, we already know that det $X$ is an invariant of (1.1), since the latter's equivalence with the isospectral system (1.2) implies that the product of the eigenvalues of $X$ is constant. This, of course, does not mean that all invariants implied by isospectrality are Casimirs. For example, letting $c(X)=\operatorname{tr} X$, we readily have

$$
\sum_{(r, s) \in \mathbb{J}_{n}} S_{(p, q),(r, s)} \frac{\partial c(X)}{\partial x_{r, s}}=\sum_{r=1}^{n} S_{(p, q),(r, r)}=\sum_{r=1}^{n}\left(n_{p, r} x_{q, r}+n_{q, r} x_{p, r}\right)=[N, X]_{p, q},
$$

which cannot be expected to vanish for all $(p, q) \in \mathrm{J}_{n}$.

Needless to say, we do not claim that $c_{1}$ and $c_{2}$ are the only Casimirs of (1.1). The full resolution of integrability, Casimirs, and other invariants of the system are deferred to Bloch et al. [4].

\section{Lie-Algebraic Representation and Its Computation}

We proved in Section 2 that $\left\{c_{(p, q),(r, s)}^{(k, l)}:(p, q),(r, s),(k, l) \in \mathrm{J}_{n}\right\}$ obey (2.2) and (2.6), hence are structure constants of an $m$-dimensional algebra $\mathfrak{g}$. This algebra 
is defined formally as a free Lie algebra

$$
\mathfrak{g}=\operatorname{FLA}\left(E_{p, q}:(p, q) \in \mathrm{J}_{n}\right),
$$

where $E_{p, q}$ are (for the time being) purely formal letters, equipped with the commutation relation

$$
\begin{aligned}
{\left[E_{p, q}, E_{r, s}\right]=} & \frac{1}{2}\left(n_{p, r} E_{q, s}+n_{p, s} E_{p, s} E_{q, r}+n_{q, r} E_{p, s}+n_{q, s} E_{p, r}\right), \\
& (p, q),(r, s) \in \mathrm{J}_{n},
\end{aligned}
$$

(see (2.5) or (2.7)). In other words, elements of $\mathfrak{g}$ are linear combinations of the $E_{p, q}$ 's (which are presumed linearly independent) and its closure under commutation is assured by (4.2).

\subsection{The Algorithm}

We recall from Section 1 that our ultimate goal it to construct representations of $\mathfrak{g}$ and of $\mathfrak{g}^{*}$ which are orthogonal to each other with respect to the Frobenius inner product $\langle Y, Z\rangle=\operatorname{tr}\left(Y^{\top} Z\right)$. Note, however, that once we determine a faithful representation of $\mathfrak{g}$ which is orthogonal-for every $(p, q),(r, s) \in \mathrm{J}_{n},\left\langle E_{p, q}, E_{r, s}\right\rangle$ is zero if $(p, q) \neq(r, s)$ and $\pi_{p, q}>0$ if $(p, q)=(r, s)$-we may take $F_{p, q}=$ $\pi_{p, q}^{-1} E_{p, q}$. This follows at once from the Riesz representation theorem for linear functionals once we observe that $\langle\cdot, \cdot\rangle$ is the standard Euclidean vector inner product, hence defining a Hilbert space.

Although, as mentioned in Section 1, the existence of a faithful representation of $\mathfrak{g}$ is assured by the Ado theorem, the latter does not provide us with specific linearly independent matrices $E_{p, q}$ (without fear of confusion, we use the same notation for formal words in (4.1) and for their representation). This motivates the work of this section, central to the entire paper. We consider the problem of finding a faithful representation of (4.1) as an exercise in numerical algebra and present an algorithm to this end.

Let us assume first that $\|N\|=1$, where $\|\cdot\|$ is the standard Euclidean matrix norm. Clearly, we may exclude the trivial case $N=O$, hence our assumption is merely a time-reparametrization of the system (1.6): Once we find a representation subject to $\|N\|=1$, we can generalize it immediately for any $N \neq O$, multiplying each $E_{p, q}$ in (4.1) by $\|N\|$.

Proposition 5. The Hermitian matrix $I+\mathrm{i} N$ is positive semidefinite and singular.

Proof. The proposition follows at once from the observation that $\sigma(I+\mathrm{i} N)=$ $1+\mathrm{i} \sigma(N), \sigma(N) \subset \mathrm{i}$, and $\rho(N)=\|N\|=1$, where $\sigma$ and $\rho$ denote the spectrum and the spectral radius, respectively. 
We seek an $n \times n$ complex upper-triangular matrix $R$ such that

$$
R^{*} R=I+\mathrm{i} N
$$

and which is in a standard form: For each row $k$ the first nonzero term (if any) is $R_{k, i_{k}}>0$, where the $i_{k}$ 's form a strictly monotone sequence, and all zero rows are at the bottom. Note that Proposition 5 implies that the bottom row of $R$ is necessarily composed of zeros. (The term "standard form" is borrowed from the usual terminology of QR factorization (Golub and van Loan [13]).) Precise details of how to compute $R$ are deferred to the next subsection.

We now remove the bottom row of $R$ and denote the new matrix by $\tilde{R}$. If $R$ has more rows of zeros, we excise them as well but in the sequel we treat merely the generic case when $\tilde{R}$ is $(n-1) \times n-$ an extension to the general case is trivial. Let

$$
B=\operatorname{Re} \tilde{R}, \quad C=\operatorname{Im} \tilde{R} .
$$

Moreover, we set

$$
A=\left[\begin{array}{l}
B \\
C
\end{array}\right]
$$

and denote its columns by $\boldsymbol{a}_{1}, \boldsymbol{a}_{2}, \ldots, \boldsymbol{a}_{n} \in \mathrm{R}^{2 n-2}$.

Proposition 6. The following is true:

$$
\begin{aligned}
B^{\top} B+C^{\top} C & =I, \\
B^{\top} C-C^{\top} B & =N, \\
\boldsymbol{a}_{p}^{\top} \boldsymbol{a}_{q} & =\delta_{p, q}, \quad p, q=1,2, \ldots, n, \\
\boldsymbol{a}_{p}^{\top} J \boldsymbol{a}_{q} & =n_{p, q}, \quad p, q=1,2, \ldots, n,
\end{aligned}
$$

where $J=\left[\begin{array}{cc}O & I \\ -I & O\end{array}\right]$ is the standard $(2 n-2) \times(2 n-2)$ symplectic matrix.

Proof. Recalling the definition of $R$,

$$
\tilde{R}^{*} \tilde{R}=R^{*} R=I+\mathrm{i} N .
$$

On the other hand, $\tilde{R}=B+\mathrm{i} C$ implies that

$$
\tilde{R}^{*} \tilde{R}=\left(B^{\top}-\mathrm{i} C^{\top}\right)(B+\mathrm{i} C)=\left(B^{\top} B+C^{\top} C\right)+\mathrm{i}\left(B^{\top} C-C^{\top} B\right) .
$$

Taking real and imaginary parts proves (4.3) and (4.4), respectively.

To prove (4.5) and (4.6), we denote the columns of $B$ and $C$ by $\boldsymbol{b}_{1}, \ldots, \boldsymbol{b}_{n} \in$ $\mathrm{R}^{n-1}$ and $\boldsymbol{c}_{1}, \ldots, \boldsymbol{c}_{n} \in \mathrm{R}^{n-1}$, respectively, and compute directly while exploiting (4.3) and (4.4):

$$
\boldsymbol{a}_{p}^{\top} \boldsymbol{a}_{q}=\left[\begin{array}{ll}
\boldsymbol{b}_{p}^{\top} & \boldsymbol{c}_{p}^{\top}
\end{array}\right]\left[\begin{array}{l}
\boldsymbol{b}_{q} \\
\boldsymbol{c}_{q}
\end{array}\right]=\left(\boldsymbol{b}_{p}^{\top} \boldsymbol{b}_{q}+\boldsymbol{c}_{p}^{\top} \boldsymbol{c}_{q}\right)=\left(B^{\top} B+C^{\top} C\right)_{p, q}=\delta_{p, q}
$$


and

$\boldsymbol{a}_{p}^{\top} J \boldsymbol{a}_{q}=\left[\begin{array}{ll}\boldsymbol{b}_{p}^{\top} & \boldsymbol{c}_{p}^{\top}\end{array}\right]\left[\begin{array}{cc}O & I \\ -I & O\end{array}\right]\left[\begin{array}{l}\boldsymbol{b}_{q} \\ \boldsymbol{c}_{q}\end{array}\right]=\boldsymbol{b}_{p}^{\top} \boldsymbol{c}_{q}-\boldsymbol{c}_{p}^{\top} \boldsymbol{b}_{q}=\left(B^{\top} C-C^{\top} B\right)_{p, q}=n_{p, q}$.

Theorem 7. Let

$$
E_{p, q}=\left(\boldsymbol{a}_{p} \boldsymbol{a}_{q}^{\top}+\boldsymbol{a}_{q} \boldsymbol{a}_{p}^{\top}\right) J, \quad(p, q) \in J_{n} .
$$

Then

(a) The set $\left\{E_{p, q}:(p, q) \in J_{n}\right\}$ obeys the commutation relations (4.2) and therefore is a representation of the Lie algebra $\mathfrak{g}$.

(b) The above representation is faithful and, moreover,

$$
\operatorname{tr} E_{p, q}^{\top} E_{r, s}= \begin{cases}\frac{1}{2}, & p=q=r=s \\ 1, & (p, q)=(r, s), p<q, \quad(p, q),(r, s) \in J_{n} . \\ 0, & (p, q) \neq(r, s),\end{cases}
$$

Proof. We prove (a) by direct computation, repeatedly using (4.6) as necessary. Let $(p, q),(r, s) \in \mathrm{J}_{n}$. Then, using the skew-symmetry of $N$,

$$
\begin{aligned}
{\left[E_{p, q}, E_{r, s}\right]=} & \frac{1}{4}\left[\left(\boldsymbol{a}_{p} \boldsymbol{a}_{q}^{\top}+\boldsymbol{a}_{q} \boldsymbol{a}_{p}^{\top}\right) J,\left(\boldsymbol{a}_{r} \boldsymbol{a}_{s}^{\top}+\boldsymbol{a}_{s} \boldsymbol{a}_{r}^{\top}\right) J\right] \\
= & \frac{1}{4}\left\{\left(\boldsymbol{a}_{q}^{\top} J \boldsymbol{a}_{r}\right) \boldsymbol{a}_{p} \boldsymbol{a}_{s}^{\top}+\left(\boldsymbol{a}_{p}^{\top} J \boldsymbol{a}_{r}\right) \boldsymbol{a}_{q} \boldsymbol{a}_{s}^{\top}+\left(\boldsymbol{a}_{q}^{\top} J \boldsymbol{a}_{s}\right) \boldsymbol{a}_{p} \boldsymbol{a}_{r}^{\top}\right. \\
& +\left(\boldsymbol{a}_{p}^{\top} J \boldsymbol{a}_{s}\right) \boldsymbol{a}_{q} \boldsymbol{a}_{r}^{\top}-\left(\boldsymbol{a}_{s}^{\top} J \boldsymbol{a}_{p}\right) \boldsymbol{a}_{r} \boldsymbol{a}_{q}^{\top}-\left(\boldsymbol{a}_{r}^{\top} J \boldsymbol{a}_{p}\right) \boldsymbol{a}_{s} \boldsymbol{a}_{q}^{\top} \\
& \left.-\left(\boldsymbol{a}_{s}^{\top} J \boldsymbol{a}_{q}\right) \boldsymbol{a}_{r} \boldsymbol{a}_{p}^{\top}-\left(\boldsymbol{a}_{r}^{\top} J \boldsymbol{a}_{q}\right) \boldsymbol{a}_{s} \boldsymbol{a}_{p}^{\top}\right\} J \\
= & \frac{1}{4}\left\{n_{p, r}\left(\boldsymbol{a}_{q} \boldsymbol{a}_{s}^{\top}+\boldsymbol{a}_{s} \boldsymbol{a}_{q}^{\top}\right)+n_{p, s}\left(\boldsymbol{a}_{q} \boldsymbol{a}_{r}^{\top}+\boldsymbol{a}_{r} \boldsymbol{a}_{q}^{\top}\right)+n_{q, r}\left(\boldsymbol{a}_{p} \boldsymbol{a}_{s}^{\top}+\boldsymbol{a}_{s} \boldsymbol{a}_{p}^{\top}\right)\right. \\
& \left.+n_{q, s}\left(\boldsymbol{a}_{p} \boldsymbol{a}_{r}^{\top}+\boldsymbol{a}_{r} \boldsymbol{a}_{p}^{\top}\right)\right\} J \\
= & \frac{1}{2}\left(n_{p, r} E_{q, s}+n_{p, s} E_{q, r}+n_{q, r} E_{p, s}+n_{q, s} E_{p, r}\right) .
\end{aligned}
$$

This confirms that the $E_{p, q}$ 's obey (4.2) and thereby proves (a).

To prove part (b) of the theorem we observe that $J \in \mathrm{O}(n)$ and use (4.5):

$$
\begin{aligned}
\operatorname{tr} E_{p, q}^{\top} E_{r, s} & =\operatorname{tr} E_{p, q} E_{r, s}^{\top}=\frac{1}{4} \operatorname{tr}\left[\left(\boldsymbol{a}_{p} \boldsymbol{a}_{q}^{\top}+\boldsymbol{a}_{q} \boldsymbol{a}_{p}^{\top}\right) J J^{\top}\left(\boldsymbol{a}_{r} \boldsymbol{a}_{s}^{\top}+\boldsymbol{a}_{s} \boldsymbol{a}_{r}^{\top}\right)\right] \\
& =\frac{1}{4} \operatorname{tr}\left[\left(\boldsymbol{a}_{p} \boldsymbol{a}_{q}^{\top}+\boldsymbol{a}_{q} \boldsymbol{a}_{p}^{\top}\right)\left(\boldsymbol{a}_{r} \boldsymbol{a}_{s}^{\top}+\boldsymbol{a}_{s} \boldsymbol{a}_{r}^{\top}\right)\right] \\
& =\frac{1}{4} \operatorname{tr}\left[\left(\boldsymbol{a}_{q}^{\top} \boldsymbol{a}_{r}\right) \boldsymbol{a}_{p} \boldsymbol{a}_{s}^{\top}+\left(\boldsymbol{a}_{q}^{\top} \boldsymbol{a}_{s}\right) \boldsymbol{a}_{p} \boldsymbol{a}_{r}^{\top}+\left(\boldsymbol{a}_{p}^{\top} \boldsymbol{a}_{r}\right) \boldsymbol{a}_{q} \boldsymbol{a}_{s}^{\top}+\left(\boldsymbol{a}_{p}^{\top} \boldsymbol{a}_{s}\right) \boldsymbol{a}_{q} \boldsymbol{a}_{r}^{\top}\right] \\
& =\frac{1}{2}\left(\delta_{p, r} \delta_{q, s}+\delta_{p, s} \delta_{q, r}\right),
\end{aligned}
$$

where we have used the identity $\operatorname{tr} \boldsymbol{a}_{k} \boldsymbol{a}_{l}^{\top}=\boldsymbol{a}_{k}^{\top} \boldsymbol{a}_{l}$. This proves (4.8). 
Since the $E_{p, q}$ 's form an orthogonal set (with respect to the Frobenius norm), they are in particular linearly independent. Therefore the representation is faithful.

\subsection{The Computation of $R$}

We have used just a single feature of the matrix $R$, namely that $R^{*} R=I+\mathrm{i} N$. (The fact that the bottom row is zero and, indeed, that all zero rows are at the bottom of the matrix, is helpful in deriving smaller matrices but not required for the construction of the faithful representation at the first place. Hence, at least in principle, we may abandon the requirement that $R$ is in a standard form, reconciling ourselves to larger matrices.) One procedure that is assured to produce $R$ in a standard form is the $Q R$ factorization $Q R$ of the Hermitian, positive semidefinite, singular matrix $(I+\mathrm{i} N)^{1 / 2}$, where $Q \in \mathrm{U}(n)$ and $R$ is upper-triangular and in a standard form. Once we abandon the requirement that $R$ is upper-triangular, hence reconciling ourselves to somewhat larger matrices, an alternative is a polar decomposition $I+\mathrm{i} N=Q R$, where $Q \in \mathrm{U}(n)$ and $R$ is Hermitian and positive semidefinite. However, the most promising route is the Cholesky factorization, which factorizes a positive definite matrix into the product $R^{*} R$, where $R$ is upper-triangular with real, positive diagonal.

We note in passing that we can replace $R$ at will by $P R$, where $P \in \mathrm{U}(n)$. (Indeed, it is trivial that the manifold of all $n \times n$ complex matrices $R$ such that $R^{*} R=I+\mathrm{i} N$ is acted multiplicatively from the left by $\mathrm{U}(n)$.) Alternatively, we can bypass $R$ altogether and construct a matrix $A$, with $n$ columns and an even number of columns, such that

$$
A^{\top} A=I, \quad A^{\top} J A=N
$$

except that this is not necessarily the easiest route.

There exist perfectly good routines (e.g., LINPAKC's CCHDC and NAG's f01bnc) that produce the Cholesky factorization of a Hermitian positive definite matrix, and they are based on a well-known comprehensive theory (Wilkinson and Reinsch [24]). Moreover, the LINPAKC (a complex extension of LINPACK) routine works with positive semidefinite matrices. Yet, there is a problem with standard Cholesky factorization in our setting. As long as the underlying Hermitian matrix is positive definite, $R$ is by definition upper-triangular with positive elements along the diagonal. If it is of rank $p, p \leq n-1$, and its principal $p \times p$ minor is nonsingular, the algorithm produces a matrix $R$ with $n-p$ zero rows at the bottom and, otherwise, the diagonal elements are positive. However, if the rank is $p \leq n-1$ but the principal $p \times p$ minor is singular, a Cholesky factorization requires pivoting and it is no longer true that $R^{*} R=I+\mathrm{i} N$.

We wish the best of both worlds: both $R$ in a standard form and a Choleskylike algorithm, that produces $R$ without any need to compute square roots. This is provided by the following straightforward extension of the Cholesky algorithm. 
We progress successively forming the $k$ th row of $R$ for $k=1,2, \ldots, p$, where $p=\operatorname{rank}(I+\mathrm{i} N) \leq n-1$.

Let $M=N$. Supposing that the first $k-1$ rows of $R$ are available, we note that

$$
\sum_{j=1}^{k}\left|r_{j, k}\right|^{2}=\sum_{j=1}^{n}\left|r_{j, k}\right|^{2}=1
$$

Thus,

$$
\sum_{j=1}^{k-1}\left|r_{j, k}\right|^{2} \leq 1
$$

If the inequality above is sharp, we let

$$
r_{k, k}=\left(1-\sum_{j=1}^{k-1}\left|r_{j, k}\right|^{2}\right)^{1 / 2}>0
$$

and set

$$
r_{k, l}=\frac{1}{r_{k, k}}\left(\mathrm{i} m_{k, l}-\sum_{j=1}^{k-1} \bar{r}_{j, k} r_{j, l}\right), \quad l=k+1, k+2, \ldots, n
$$

It is trivial to confirm that the $k$ th row of $R$ is consistent with $R^{*} R=I+\mathrm{i} M$.

If there is an equality in (4.9) we replace $R$ with $R P_{k}$, where $P_{k}$ is the permutation matrix that cycles the columns $k, k+1, \ldots, n$ to $k+1, k+2, \ldots, n, k$ and, simultaneously, replace $M$ by $P_{k}^{\top} M P_{k} \in \mathfrak{s} o(n)$. Form the new sum $\sum_{j=1}^{k-1}\left|r_{j, k}\right|^{2}$ : if it is strictly less than one, continue as above, otherwise continue cycling. We can cycle at most $n-k$ times. If the sum equals one in each case, then necessarily $\operatorname{rank}(I+\mathrm{i} N)=k$ and we can pad the bottom $n-k$ rows of $R$ with zeros.

The outcome of this procedure is an upper-triangular matrix $R$ and a product $\hat{P}$ of permutation matrices, such that $R^{*} R=I+\mathrm{i} M=\hat{P}^{\top}(I+\mathrm{i} N) \hat{P}$. We finally replace $R$ by $R \hat{P}^{\top}$, whereby $R^{*} R=I+\mathrm{i} N$, as required. Note that, unlike as in standard Cholesky factorization, it is entirely possible that elements of $R$ vanish along the diagonal, but that the matrix is in a standard form.

We mention in passing an obvious amendment to the above procedure, full row pivoting of $R$ : instead of cycling the $k, k+1, \ldots, n$ columns when $r_{k, k}$ is zero, we exchange columns in each step so that the new pivot element $r_{k, k}$ is the largest possible. For large dimensions this procedure has the virtue of better stability.

As an example of our algorithm, consider

$$
N=\left[\begin{array}{rrrr}
0 & 1 & 0 & 0 \\
-1 & 0 & 0 & 0 \\
0 & 0 & 0 & 0 \\
0 & 0 & 0 & 0
\end{array}\right]
$$


and note that $\|N\|=1$. For $k=1$ we obtain $r_{1,1}=1, r_{1,2}=\mathrm{i}$ and $r_{1,3}=r_{1,4}=0$, but $k=2$ results in an equality in (4.9). We thus let

$$
M \leftarrow P_{2}^{\top} M P_{2}=\left[\begin{array}{rrrr}
0 & 0 & 0 & \mathrm{i} \\
0 & 0 & 0 & 0 \\
0 & 0 & 0 & 0 \\
-\mathrm{i} & 0 & 0 & 0
\end{array}\right], \quad R=\left[\begin{array}{cccc}
1 & 0 & 0 & \mathrm{i} \\
0 & \times & \times & \times \\
0 & \times & \times & \times \\
0 & \times & \times & \times
\end{array}\right] .
$$

Now (4.9) is sharp and $r_{2,2}=1, r_{2,3}=r_{2,4}=0$. Likewise, for $k=3$ we have $r_{3,3}=1, r_{3,4}=0$. Finally, for $k=4$ we have a row of zeros. Since $\hat{P}=P_{2}$, we finally set

$$
R \leftarrow R \hat{P}=\left[\begin{array}{llll}
1 & \mathrm{i} & 0 & 0 \\
0 & 0 & 1 & 0 \\
0 & 0 & 0 & 1 \\
0 & 0 & 0 & 0
\end{array}\right]
$$

in a standard form. It is trivial that, indeed, $R^{*} R=I+\mathrm{i} N$.

\subsection{An Example}

Let

$$
N=\left[\begin{array}{rrr}
0 & a & b \\
-a & 0 & c \\
-b & -c & 0
\end{array}\right]
$$

where $a, b, c \in \mathrm{R}, a^{2}+b^{2}+c^{2}=1$. It is easy to check that $\|N\|=1$. Assuming for simplicity that $|a|<1$, the procedure of Subsection 4.2 yields

$$
R=\left[\begin{array}{ccc}
1 & \mathrm{i} a & \mathrm{i} b \\
0 & \sqrt{b^{2}+c^{2}} & \frac{-a b+\mathrm{i} c}{\sqrt{b^{2}+c^{2}}} \\
0 & 0 & 0
\end{array}\right]
$$

Once we excise the bottom row, we obtain

$$
A=\left[\begin{array}{ccc}
1 & 0 & 0 \\
0 & \sqrt{b^{2}+c^{2}} & -\frac{a b}{\sqrt{b^{2}+c^{2}}} \\
0 & a & b \\
0 & 0 & \frac{c}{\sqrt{b^{2}+c^{2}}}
\end{array}\right],
$$

consequently, by (4.7),

$$
E_{1,1}=\left[\begin{array}{cccc}
0 & 0 & 1 & 0 \\
0 & 0 & 0 & 0 \\
0 & 0 & 0 & 0 \\
0 & 0 & 0 & 0
\end{array}\right]
$$




$$
\begin{aligned}
& E_{1,2}=\left[\begin{array}{cccc}
-\frac{1}{2} a & 0 & 0 & \frac{1}{2} \sqrt{b^{2}+c^{2}} \\
0 & 0 & \frac{1}{2} \sqrt{b^{2}+c^{2}} & 0 \\
0 & 0 & \frac{1}{2} a & 0 \\
0 & 0 & 0 & 0
\end{array}\right], \\
& E_{1,3}=\left[\begin{array}{cccc}
-\frac{1}{2} b & -\frac{c}{2 \sqrt{b^{2}+c^{2}}} & 0 & -\frac{a b}{2 \sqrt{b^{2}+c^{2}}} \\
0 & 0 & -\frac{a b}{2 \sqrt{b^{2}+c^{2}}} & 0 \\
0 & 0 & \frac{1}{2} b & 0 \\
0 & 0 & \frac{c}{2 \sqrt{b^{2}+c^{2}}} & 0
\end{array}\right] \text {, } \\
& E_{2,2}=\left[\begin{array}{cccc}
0 & 0 & 0 & 0 \\
-a \sqrt{b^{2}+c^{2}} & 0 & 0 & b^{2}+c^{2} \\
-a^{2} & 0 & 0 & a \sqrt{b^{2}+c^{2}} \\
0 & 0 & 0 & 0
\end{array}\right] \text {, } \\
& E_{2,3}=\left[\begin{array}{cccc}
0 & 0 & 0 & 0 \\
\frac{b\left(a^{2}-b^{2}-c^{2}\right)}{2 \sqrt{b^{2}+c^{2}}} & -\frac{1}{2} c & 0 & -a b \\
-a b & -\frac{a c}{2 \sqrt{b^{2}+c^{2}}} & 0 & -\frac{b\left(a^{2}-b^{2}-c^{2}\right)}{2 \sqrt{b^{2}+c^{2}}} \\
-\frac{a c}{2 \sqrt{b^{2}+c^{2}}} & 0 & 0 & \frac{1}{2} c
\end{array}\right] \text {, } \\
& E_{3,3}=\left[\begin{array}{cccc}
0 & 0 & 0 & 0 \\
\frac{a b^{2}}{\sqrt{b^{2}+c^{2}}} & \frac{a b c}{b^{2}+c^{2}} & 0 & \frac{a^{2} b^{2}}{b^{2}+c^{2}} \\
-b^{2} & -\frac{b c}{\sqrt{b^{2}+c^{2}}} & 0 & -\frac{a b^{2}}{\sqrt{b^{2}+c^{2}}} \\
-\frac{b c}{\sqrt{b^{2}+c^{2}}} & -\frac{c^{2}}{b^{2}+c^{2}} & 0 & -\frac{a b c}{b^{2}+c^{2}}
\end{array}\right] \text {. }
\end{aligned}
$$

The above basis does not share the symmetry implicit in reversing the order of rows and columns of $N$. This is hardly an impediment, except perhaps on æsthetic grounds, but we note as a matter of interest that, at least for $n=3$, we can single out a "symmetric" basis,

$$
E_{1,1}=\left[\begin{array}{cccc}
0 & \frac{1}{2}(a+c) & \frac{1}{2}(a-c) & b \\
0 & 0 & 0 & 0 \\
0 & 0 & 0 & 0 \\
0 & 0 & 0 & 0
\end{array}\right]
$$




$$
\begin{aligned}
& E_{1,2}=\left[\begin{array}{rccc}
-\frac{1}{2} a & -\frac{1}{2} b & \frac{1}{2} b & \frac{1}{2} c \\
0 & \frac{1}{4}(a+c) & \frac{1}{4}(a-c) & \frac{1}{2} b \\
0 & \frac{1}{4}(a+c) & \frac{1}{4}(a-c) & \frac{1}{2} b \\
0 & 0 & 0 & 0
\end{array}\right], \\
& E_{1,3}=\left[\begin{array}{rccc}
-\frac{1}{2} b & \frac{1}{4}(a-c) & -\frac{1}{4}(a+c) & 0 \\
0 & 0 & 0 & 0 \\
0 & 0 & 0 & 0 \\
0 & \frac{1}{4}(a+c) & \frac{1}{4}(a-c) & \frac{1}{2} b
\end{array}\right] \text {, } \\
& E_{2,2}=\left[\begin{array}{rrrr}
0 & 0 & 0 & 0 \\
-a & -b & b & c \\
-a & -b & b & c \\
0 & 0 & 0 & 0
\end{array}\right] \\
& E_{2,3}=\left[\begin{array}{cccc}
0 & 0 & 0 & 0 \\
-\frac{1}{2} b & \frac{1}{4}(a-c) & -\frac{1}{4}(a+c) & 0 \\
-\frac{1}{2} b & \frac{1}{4}(a-c) & -\frac{1}{4}(a+c) & 0 \\
-\frac{1}{2} a & -\frac{1}{2} b & \frac{1}{2} b & \frac{1}{2} c
\end{array}\right] \\
& E_{3,3}=\left[\begin{array}{cccc}
0 & 0 & 0 & 0 \\
0 & 0 & 0 & 0 \\
0 & 0 & 0 & 0 \\
-b & \frac{1}{2}(a-c) & -\frac{1}{2}(a+c) & 0
\end{array}\right] \text {, }
\end{aligned}
$$

which is consistent with the commutation relations (4.2).

We mention in passing that it is possible — not by our algorithm, though — to derive unfaithful representations for sufficiently sparse matrices $N$. For example, for

$$
N=\left[\begin{array}{rrr}
0 & 1 & 0 \\
-1 & 0 & 0 \\
0 & 0 & 0
\end{array}\right]
$$

we might take

$$
\begin{aligned}
E_{1,1}=\left[\begin{array}{ccc}
-\frac{\sqrt{2}}{4} & -\frac{1}{2}-\frac{\sqrt{2}}{4} & 0 \\
\frac{1}{2}-\frac{\sqrt{2}}{4} & \frac{\sqrt{2}}{4} & 0 \\
0 & 0 & 0
\end{array}\right], & E_{1,2}=\left[\begin{array}{ccc}
-\frac{\sqrt{2}}{4} & \frac{\sqrt{2}}{4} & 0 \\
\frac{\sqrt{2}}{4} & \frac{\sqrt{2}}{4} & 0 \\
0 & 0 & 0
\end{array}\right], \\
E_{1,3}=\left[\begin{array}{ccc}
0 & 0 & -1-\sqrt{2} \\
0 & 0 & 1 \\
0 & 0 & 0
\end{array}\right], & E_{2,2}=\left[\begin{array}{ccc}
\frac{\sqrt{2}}{4} & -\frac{1}{2}+\frac{\sqrt{2}}{4} & 0 \\
\frac{1}{2}+\frac{\sqrt{2}}{4} & -\frac{\sqrt{2}}{4} & 0 \\
0 & 0 & 0
\end{array}\right],
\end{aligned}
$$




$$
E_{2,3}=\left[\begin{array}{ccc}
0 & 0 & 1 \\
0 & 0 & 1+\sqrt{2} \\
0 & 0 & 0
\end{array}\right], \quad E_{3,3}=\left[\begin{array}{lll}
0 & 0 & 0 \\
0 & 0 & 0 \\
0 & 0 & 0
\end{array}\right] .
$$

Clearly, $E_{3,3}=O$ and the representation is not faithful. This, at the very least, demonstrates the care that must be exercised in the construction of representations for general $N$, since the absence of faithfulness might often be less obvious.

\section{Concluding Remarks}

Having commenced from equation (1.1), we proved that it is endowed with a Poisson structure and investigated invariants. Because of the relationship of Poisson structures with flows along orbits in the dual to the corresponding Lie algebra $\mathfrak{g}$, we have explored the issue of faithful representations and their numerical generation.

The problem of the faithful representation of a Lie algebra has been treated in symbolic algebra and de Graff [9] has provided a very interesting algorithm. Yet, the very generality of this algorithm is its downfall in specific applications, since it produces representations of very large size. Ideally, we strive for minimal representations, in practice we are willing to compromise on "tight" representations. In Section 4 we have presented an algorithm that generates faithful representations for commutation relations (4.2). Clearly, the algorithm is application-specific and it is unlikely that a similar approach can be applied to a wider (or a different) set of structure constants.

The construction of faithful Lie-algebraic representations has never, to the best of our knowledge, been considered in a numerical-algebraic setting. We firmly believe that, insofar as "tight" representations are concerned, this is the right way forward. In the course of our research into more general isospectral flows and Poisson systems, we have assembled a significant collection of structure constants that obey the Jacobi identity. Only in a few cases are their faithful representations known. A common thread running through all the cases when we have managed to identify a faithful representation is that matrices are low-rank (in our case they are of rank 2). It is premature to hypothesize whether this represents a valid general approach.

\section{Acknowledgments}

We would like to thank Brad Baxter (Birkbeck College), Matania Ben Artzi (Hebrew University), Roger Brockett (Harvard), John Butcher (University of Auckland), Willem de Graff (University of Linz), Adi Levin (Cadent), David Levin (University of Tel Aviv), Marta Mazzocco (University Manchester), Hans MuntheKaas (University of Bergen), Peter Olver (University of Minnesota), Gopal Prasad (University of Michigan), Tudor Ratiu (EPFL Lausanne), and Jeremy Schiff (Bar Ilan University). The work of AMB was supported by the National Science Foun- 
dation, while the work of AI was performed in part as a visitor at the University of Tel Aviv.

\section{References}

[1] A. M. Bloch and A. Iserles, On the optimality of double-bracket flows, Int. J. Math. Math. Sci. 15 (2005), 793-801.

[2] A. M. Bloch, R. W. Brockett, and P. E. Crouch, Double bracket equations and geodesic flows on symmetric spaces, Comm. Math. Phys. 187 (1997), 357-373.

[3] A. M. Bloch, R. W. Brockett, and T. S. Ratiu, Completely integrable gradient flows, Comm. Math. Phys. 147 (1992), 57-74.

[4] A. M. Bloch, A. Iserles, J. E. Marsden, and T. S. Ratiu, A class of integrable geodesic flows on the symplectic group, Technical Report, University of Cambridge. To appear.

[5] R. W. Brockett, Dynamical systems that sort lists, diagonalize matrices, and solve linear programming problems, Linear Algebra Appl. 146 (1991), 79-91.

[6] M. T. Chu, Matrix differential equations: A continuous realization process for linear algebra problems, Nonlinear Anal. 18 (1992), 1125-1146.

[7] M. T. Chu, A list of matrix flows with applications, in Hamiltonian and Gradient Flows, Algorithms and Control, Fields Inst. Commun., No. 3, Amer. Math. Soc., Providence, RI, 1994, pp. 87-97.

[8] M. T. Chu and K. R. Driessel, Constructing symmetric nonnegative matrices with prescribed eigenvalues by differential equations, SIAM J. Math. Anal. 22 (1991), 1372-1387.

[9] W. A. de Graff, Constructing faithful matrix representations of Lie algebras, in Proceedings of the 1997 International Symposium on Symbolic and Algebraic Computation (Kihei, HI), 1997, pp. 54-59.

[10] P. Deift, T. Nanda, and C. Tomei, Ordinary differential equations and the symmetric eigenvalue problem, SIAM J. Numer. Anal. 20 (1983), 1-22.

[11] K. Eng $\varnothing$ and S. Faltinsen, Numerical integration of Lie-Poisson systems while preserving coadjoint orbits and energy, SIAM J. Numer. Anal. 39 (2001), 128-145.

[12] H. Flaschka, The Toda lattice, Phys. Rev. B 9 (1974), 1924-1925.

[13] G. H. Golub and C. F. van Loan, Matrix Computations, 2nd ed., The Johns Hopkins University Press, Baltimore, 1989.

[14] R. A. Horn and C. R. Johnson, Topics in Matrix Analysis, Cambridge University Press, Cambridge, 1991.

[15] D. Lewis and J. C. Simo, Conserving algorithms for the dynamics of Hamiltonian systems on Lie groups, J. Nonlinear Sci. 4 (1994), 253-299.

[16] S. V. Manakov, Note on the integration of Euler's equations of the dynamics of an $n$-dimensional rigid body, Funct. Anal. Appl. 10 (1976), 328-329.

[17] J. E. Marsden and T. S. Ratiu, (1994), Introduction to Mechanics and Symmetry, Springer-Verlag, New York.

[18] P. J. Olver, (1995), Equivalence, Invariants, and Symmetry, Cambridge University Press, Cambridge.

[19] T. Ratiu, The motion of the free $n$-dimensional rigid body, Indiana Univ. Math. J. 29 (1980), 609-627.

[20] W. W. Symes, The QR algorithm and scattering for the finite nonperiodic Toda lattice, Phys. $D$ 4 (1981/82), 275-280.

[21] M. Toda, Theory of Nonlinear Lattices, 2nd ed., Springer-Verlag, Berlin, 1987.

[22] V. S. Varadarajan, Lie Groups, Lie Algebras, and Their Representations, Springer-Verlag, Berlin, 1984.

[23] D. Watkins, Isospectral flows, SIAM Rev. 26 (1984), 379-391.

[24] J. H. Wilkinson and C. Reinsch, eds., Linear Algebra, No. II in Handbook for Automatic Computation, Springer-Verlag, Berlin, 1971. 